\section{Activating autophagy}

Strategies that promote autophagy could be beneficial in several disease settings, but as yet no specific autophagy-inducing agents have been identified. Now, a study published in Nature describes a potent autophagyinducing peptide with promising efficacy in several infectious diseases and in the clearance of protein aggregates in neurodegenerative diseases.

As microbial virulence factors often manipulate host autophagy responses, Levine and colleagues began by investigating the interaction between the HIV-1 protein Nef and the autophagy-inducing protein beclin 1 . They identified a region on beclin 1 that is necessary for its autophagic function, and then designed a peptide composed of this region linked to the HIV-1 Tat protein to promote cell permeability.

Subsequent in vitro experiments confirmed that this Tat-beclin 1 peptide is a potent inducer of autophagy. Increased autophagosome and autolysosome numbers, as well as enhanced protein degradation, were seen in peptide-treated HeLa cells. The authors also showed that it interacted with a previously unknown negative regulator of autophagy, GAPR-1 (also known as GLIPR2).

Nonspecific pharmacological activation of autophagy is known to clear aggregates of proteins with expanded polyglutamine tracts that are characteristic of several neurodegenerative diseases such as Huntington's disease. Tat-beclin 1 successfully cleared small aggregates of htt103Q (a model polyglutamine expansion protein derived from mutant human huntingtin) in HeLa cells, and reduced levels of soluble htt103Q. These results indicate the potential utility of Tat-beclin 1 in both treating and preventing the protein aggregation implicated in such diseases.

The authors also investigated the therapeutic properties of Tat-beclin 1 in vitro against the following viruses: Sindbis virus (SINV), chikungunya virus (CHIKV), West Nile virus (WNV) and HIV-1. Tat-beclin 1 treatment induced a 10-50-fold reduction in SINV, CHIKV and WNV titres in infected HeLa cells; importantly, this was not due to the cytotoxicity of the peptide. HIV-1 replication in human monocytederived macrophages was also substantially inhibited following Tat-beclin 1 treatment. In addition, the peptide decreased survival of the intracellular bacterium Listeria monocytogenes in mouse bonemarrow-derived macrophages.

Tat-beclin 1 also induced autophagy in mice and was well tolerated. Treatment with Tat-beclin 1 reduced viral titres in both neonatal CHIKV-infected mice and in neonatal WNV-infected mice, and substantially reduced mortality in both models, further demonstrating the anti-infective activity of the peptide.

Autophagy-inducing peptides such as Tat-beclin 1 could therefore represent a promising therapeutic approach for several conditions, including infectious and neurodegenerative diseases.

Mariam Faruqi

ORIGINAL RESEARCH PAPER Shoji-Kawata, S. et al. Identification of a candidate therapeutic autophagy-inducing peptide. Nature 30 Jan 2013 (doi:10.1038/nature11866)

FURTHER READING Rubinsztein, D. C. et al.

Autophagy modulation as a potential therapeutic target for diverse diseases. Nature Rev. Drug Discov. 11, 709-730 (2012)

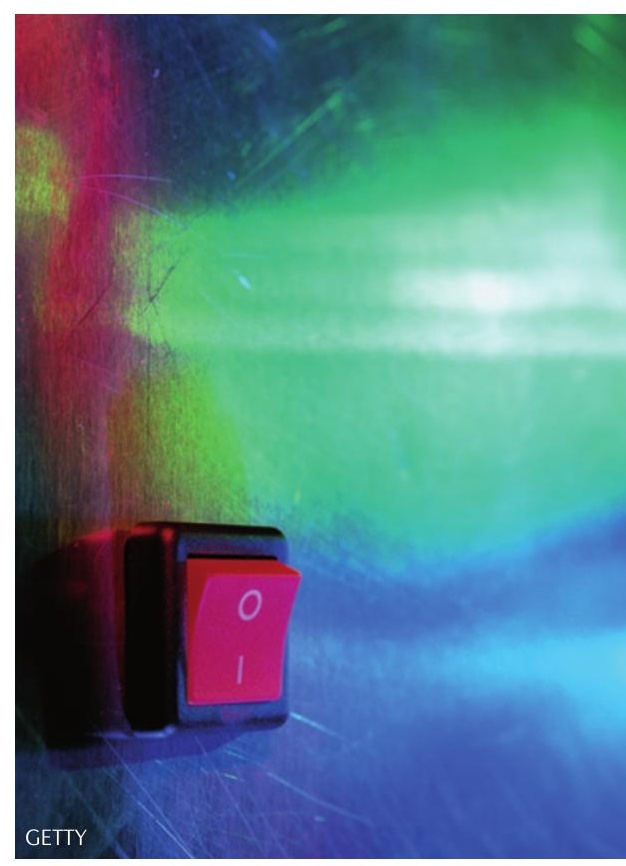

